\title{
Faddeev treatment of long-range correlations and the one-hole spectral function of ${ }^{16} \mathrm{O}$
}

\author{
C. Barbieri ${ }^{1}$ and W. H. Dickhoff ${ }^{1,2}$ \\ ${ }^{1}$ Department of Physics, Washington University, St. Louis, Missouri 63130 \\ ${ }^{2}$ Laboratory of Theoretical Physics, Ghent University, Proeftuinstraat 86, B-9000 Gent, Belgium
}

(Received 20 November 2001; published 3 June 2002)

\begin{abstract}
The Faddeev technique is employed to study the influence of both particle-particle and particle-hole phonons on the one-hole spectral function of ${ }^{16} \mathrm{O}$. Collective excitations are accounted for at a random phase approximation level and subsequently summed to all orders by the Faddeev equations to obtain the nucleon selfenergy. An iterative procedure is applied to investigate the effects of the self-consistent inclusion of the fragmentation in the determination of the phonons and the corresponding self-energy. The present results indicate that the characteristics of hole fragmentation are related to the low-lying states of ${ }^{16} \mathrm{O}$.

DOI: 10.1103/PhysRevC.65.064313

PACS number(s): 21.10.Jx, 21.60.Jz
\end{abstract}

\section{INTRODUCTION}

Correlations in the nucleus produce a substantial reduction of the occupation probability of single-particle (SP) shells with respect to the independent-particle model (IPM) prediction. A substantial part of this depletion is due to the coupling to high-lying excitations reached by the strong short-range and tensor components of the nucleon-nucleon interaction $[1,2]$. At low energy, the presence of various collective modes may result in a rearrangement of the SP strength distribution around the Fermi energy [3]. Experimentally, these features can be observed in the $\left(e, e^{\prime} p\right)$ reaction as a reduction of the absolute spectroscopic factors for the knockout of a nucleon from a valence shell, as the strong fragmentation of the spectral strength for more deeply bound SP states, and by the appearance of small fragments associated with SP states that are empty in the IPM. Studies of $\left(e, e^{\prime} p\right)$ reactions have determined absolute spectroscopic factors in many closed-shell nuclei [4-6] demonstrating that the removal probability for nucleons from these systems is reduced by about $35 \%$ with respect to the shell-model predictions. More recently, also $\left(e, e^{\prime} N N\right)$ reactions, that involve the emission of two nucleons from the target, have also become feasible $[7,8]$. The latter were motivated by the possibility to directly study the high-momentum components, produced by short-range and tensor correlations (SRTC), between pairs of nucleons at low energy. Such experiments are now able to disentangle the most relevant lowest states of the residual $A-2$ nucleus [9] which are also influenced by the presence of low-energy correlations.

The theoretical study of one- and two-hole spectral functions to understand the results of the above reactions, usually requires substantial efforts in computational many-body physics and does not always give a complete explanation of the observed data. In particular, the nucleus of ${ }^{16} \mathrm{O}$ is still not completely understood at the microscopic level and theoretical calculations of its properties still fail in two ways.

First, the experimental spectroscopic strength [10] for the knockout of a proton from both the $p_{1 / 2}$ and $p_{3 / 2}$ shells corresponds to about $60 \%$. The outgoing proton is described by a wave distorted by a complex optical potential which describes the corresponding elastic proton scattering data. This introduces an uncertainty in these spectroscopic factors since the $\left(e, e^{\prime} p\right)$ reaction probes the interior of the nucleus, where elastic proton scattering is less sensitive. The inclusion of a relativistic description of the outgoing proton in the analysis of the data can further change these results somewhat [11]. An assignment of a 5 to $10 \%$ error for the absolute spectroscopic factors appears quite reasonable at this time. Several studies have focused on the effect of short-range correlations [12-14] and computed spectroscopic factors directly for ${ }^{16} \mathrm{O}$. All these works yield a strength reduction of about $10 \%$ in general agreement with expectations based on nuclearmatter calculations [1]. Center-of-mass corrections are known to raise the spectroscopic factor by about $7 \%[15,16]$, resulting in a substantial disagreement with data. The effects of long-range correlations (LRC) were studied in Refs. $[17,18]$. The inclusion of LRC in these works is limited to the Tamm-Dancoff approximation (TDA) for the intermediate two-particle-one-hole $(2 p 1 h)$ and two-hole-oneparticle $(2 h 1 p)$ states in the nucleon self-energy using the $G$ matrix as a residual interaction. The reduction of the $p_{1 / 2}$ and $p_{3 / 2}$ spectroscopic factors due to these LRC is about $15 \%$. In the calculations of Ref. [17], a combined treatment of LRC and SRTC was obtained. The resulting $p_{1 / 2}$ and $p_{3 / 2}$ spectroscopic factors correspond to 77 and $76 \%$ of the SP strength, respectively, without inclusion of center-of-mass corrections. Short-range effects were included by employing the energy dependence of the Brueckner $G$ matrix in the corresponding self-energy contribution. The resulting disagreement with the experimental data appears to be about $15-20 \%$ but some allowance for the uncertainty of the extraction of the spectroscopic factors should be factored in. The results of Refs. $[17,18]$ clearly show that a substantial improvement can be obtained by the inclusion of LRC. Looking at the overall picture, a comparison between the quoted results suggests that the quenching produced by SRTC appears to be well established. At the same time, low-energy LRC are identified as an essential ingredient that is needed for a complete understanding of the discrepancy with the experimental data.

The second issue regarding ${ }^{16} \mathrm{O}$ concerns a satisfactory understanding of the fragmentation of the SP strength at low energy. The full one-hole spectral function for small missing energies was computed in Ref. [17] and the results were subsequently used as a starting point for the study of the ${ }^{16} \mathrm{O}\left(e, e^{\prime} p p\right)$ reaction $[19,20]$. The high-momentum compo- 
nents of the two-hole overlap function caused by short-range effects were also included by adding the proper defect functions computed directly for the ${ }^{16} \mathrm{O}$ nucleus [21]. These calculations led to a proper description of the experimental cross section for two proton emission $[7,8]$ to the ground state of ${ }^{14} \mathrm{C}$. In spite of these successes, the one- and twohole spectral functions still miss some key features of the fragmentation and do not describe all the relevant low-lying states of the residual nuclei ${ }^{15} \mathrm{~N}$ and ${ }^{14} \mathrm{C}$. In particular, the experiments show that the spectral strength for the emission of a $p_{3 / 2}$ proton is fragmented in one big peak and a few smaller ones [10]. The latter are found at a slightly higher missing energy and carry a reasonable amount of strength: of the $60 \%$ observed mean-field strength, about $5 \%$ is distributed in these small peaks while the rest is in the main fragment. This feature is not reproduced by the results of Refs. $[17,18]$, where all the strength is concentrated in one single peak. Other experimentally observed hole fragments, such as the $d_{5 / 2}$ and $s_{1 / 2}$ found at about $-17.4 \mathrm{MeV}$ missing energy, are not reproduced as well.

Analogously, the spectrum of ${ }^{14} \mathrm{C}$ contains two low-lying isovector $2^{+}$levels, that can be reached by two-nucleon emission, but only one of them is reproduced by the above theoretical calculations. This missing state represents the main discrepancy between the theoretical and experimental ${ }^{16} \mathrm{O}\left(e, e^{\prime} p p\right)$ cross sections [8]. It is interesting to note that the transition to both of the $2^{+}$states can be interpreted as the knockout of two protons from a $p_{1 / 2}$ and a $p_{3 / 2}$ state. Although this has not been directly investigated before, it is natural to suppose a connection exists between the spectrum of ${ }^{14} \mathrm{C}$ and the fragmentation of the $p_{3 / 2}$ strength in ${ }^{15} \mathrm{~N}$. The presence of a very low-lying $2^{+}$state in ${ }^{16} \mathrm{O}$ can also play a role. The fragmentation of the $p_{3 / 2}$ strength, in turn, can be interpreted as a $p_{3 / 2}$ hole on either the ground state of ${ }^{16} \mathrm{O}$ or one of its low-lying positive parity states. Since these spectra and transition amplitudes are naturally linked to each other, a formalism in which all of them are obtained in a selfconsistent way is desirable, if not necessary, to resolve the above issues. Such self-consistent calculations have so far been restricted to second-order contributions to the selfenergy using a Skyrme force for the effective interaction [22]. Such a treatment of LRC in ${ }^{16} \mathrm{O}$ is inadequate since it does not include any residual interaction between the intermediate $2 p 1 h$ and $2 h 1 p$ states in the self-energy.

The merit of the calculations of Refs. $[17,18]$ was that the interaction between the $2 p 1 h$ states (and $2 h 1 p$ states) was summed to all orders. Thus a simultaneous description of the effects of both particle-hole $(p h)$ and hole-hole $(h h)$ [as well as particle-particle $(p p)]$ collective excitations was achieved, including the interplay between them. These collective excitations, though, were accounted for only at the TDA level. The simultaneous treatment of $p h$ and $p p(h h)$ excitations is not a trivial problem and an extension of these calculations beyond TDA presents serious difficulties [23]. On the other hand, in order to account for the coupling to collective excitations that are actually observed in ${ }^{16} \mathrm{O}$ it is necessary to at least consider a random phase approximation (RPA) description of the isoscalar negative parity states [24]. To account for the low-lying isoscalar positive parity states an even more complicated treatment will be required [25]. Sizable collective effects are also present in the particleparticle $(p p)$ and $h h$ excitations involving tensor correlations for isoscalar and pair correlations for isovector states. Also in this case, an RPA treatment would be relevant.

The aim of the present paper is to extend the calculations of Ref. [17] to include such RPA correlations. A formalism has been proposed recently that allows the simultaneous inclusion of $p h$ and $p p(h h)$ excitations at the RPA level $[26,27]$. In this scheme, the RPA collective excitations can be summed to all orders by solving a set of Faddeev equations for the motion of $2 p 1 h$ and $2 h 1 p$ excitations. This is done within the framework of self-consistent Green's function theory (SCGF) in which the equations of motion are expressed in terms of the dressed (fragmented) SP propagator. The resulting self-energy contains the inclusion of these $p p(h h)$ and $p h$ RPA phonons to all orders and therefore allows for an improved description of the influence of LRC on the SP propagator. In turn, the results for the hole (and particle) spectral functions can be employed in a subsequent dressed RPA (DRPA) calculation and then iterated to investigate the effects of self-consistency on the fragmentation.

In Sec. II we describe the details of the formalism and its implementation for ${ }^{16} \mathrm{O}$. The main points of the Faddeev formalism are reviewed in Sec. II B. The results for the onehole spectral function are given in Sec. III. First, the Faddeev equations are solved including RPA phonons and a discussion of the improvements over a calculation at the TDA level is presented. Then, in Sec. III B the RPA results are iterated a few times to investigate the effects introduced by selfconsistent fragmentation. In Sec. III C, the Faddeev formalism is used to further investigate the relations between the $p h$ spectra and the SP strength with regard to some of the unresolved questions. This analysis may give further insight into the understanding of the low-lying spectra as well as hints for future calculations. Conclusions are drawn in Sec. IV.

\section{CALCULATION OF THE SINGLE-PARTICLE GREEN'S FUNCTION}

We consider the calculation of the SP Green's function

$$
g_{\alpha \beta}(\omega)=\sum_{n} \frac{\left(\mathcal{X}_{\alpha}^{n}\right)^{*} \mathcal{X}_{\beta}^{n}}{\omega-\varepsilon_{n}^{+}+i \eta}+\sum_{k} \frac{\mathcal{Y}_{\alpha}^{k}\left(\mathcal{Y}_{\beta}^{k}\right)^{*}}{\omega-\varepsilon_{k}^{-}-i \eta}
$$

from which both the one-hole and one-particle spectral functions, for the removal and addition of a nucleon, can be extracted. In Eq. (1), $\quad \mathcal{X}_{\alpha}^{n}=\left\langle\Psi_{n}^{A+1}\left|c_{\alpha}^{\dagger}\right| \Psi_{0}^{A}\right\rangle \quad\left(\mathcal{Y}_{\alpha}^{k}\right.$ $\left.=\left\langle\Psi_{k}^{A-1}\left|c_{\alpha}\right| \Psi_{0}^{A}\right\rangle\right)$ are the spectroscopic amplitudes for the excited states of a system with $A+1(A-1)$ particles and the poles $\varepsilon_{n}^{+}=E_{n}^{A+1}-E_{0}^{A}\left(\varepsilon_{k}^{-}=E_{0}^{A}-E_{k}^{A-1}\right)$ correspond to the excitation energies with respect to the $A$-body ground state. The indices $n$ and $k$ enumerate the fragments associated with the one-particle and one-hole excitations, respectively. The one-body Green's function can be computed by solving the Dyson equation 


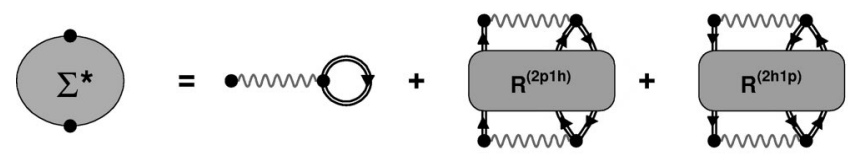

FIG. 1. Diagrams contributing to the irreducible self-energy $\Sigma *$. The double lines may represent either an IPM or a dressed propagator. The wavy lines correspond to $G$-matrix interactions. The first term is the Brueckner-Hartree-Fock potential while the others represent the $2 p 1 h / 2 h 1 p$ or higher contributions that are approximated through the Faddeev TDA/RPA equations.

$$
g_{\alpha \beta}(\omega)=g_{\alpha \beta}^{0}(\omega)+\sum_{\gamma \delta} g_{\alpha \gamma}^{0}(\omega) \Sigma_{\gamma \delta}^{*}(\omega) g_{\delta \beta}(\omega),
$$

where the irreducible self-energy $\Sigma_{\gamma \delta}^{*}(\omega)$ acts as an effective energy-dependent potential. The latter can be expanded in a Feynman-Dyson series [28,29] in terms of either the meanfield IPM propagator $g_{\alpha, \beta}^{0}(\omega)$ or even the exact propagator $g_{\alpha \beta}(\omega)$, which itself is a solution of Eq. (2). In general, $\Sigma_{\gamma \delta}^{*}(\omega)$ can be represented as the sum of a one-body Hartree-Fock potential and terms that describe the coupling between the SP motion and more complex excitations [30]. This separation is depicted by the diagrams of Fig. 1. In particular, the latter contributions can be expressed in terms of a three-line irreducible propagator $R(\omega)$ which describes the propagation of at least $2 h 1 p$ or $2 p 1 h$ at the same time. It is at the level of $R(\omega)$ that the correlations involving interactions between different collective modes need to be included.

The spectroscopic factors $Z_{k}$ for the removal of a nucleon from the $A$ particle system, while leaving the residual nucleus in its $k$ th excited state, is obtained from the spectroscopic amplitudes $\mathcal{Y}_{\alpha}^{k}$. The latter are normalized by

$$
Z_{k}=\sum_{\alpha}\left|\mathcal{Y}_{\alpha}^{k}\right|^{2}=1+\left.\sum_{\alpha, \beta}\left(\mathcal{Y}_{\alpha}^{k}\right) * \frac{\partial \Sigma_{\alpha \beta}^{*}(\omega)}{\partial \omega}\right|_{\omega=\varepsilon_{k}^{-}} \mathcal{Y}_{\beta}^{k}
$$

This result follows directly from the Dyson equation (2). The same relation applies also to the one-particle spectroscopic amplitudes $\mathcal{X}_{\alpha}^{n}$. The present calculations were performed within a finite set of harmonic oscillator states, representing the closed shells that are most relevant for low-lying excitations. As a consequence of the truncation of the model space a Brueckner $G$ matrix was employed in evaluating the diagrams of Fig. 1. The calculation of the Hartree-Fock term and the approximation of the $2 p 1 h / 2 h 1 p$ propagator are discussed in the following.

\section{A. Brueckner-Hartree-Fock self-energy}

The first diagram on the right-hand side of Fig. 1 represents the Brueckner-Hartree-Fock (BHF) contribution to the self-energy. This is given analytically by

$$
\Sigma_{\alpha \beta}^{\mathrm{BHF}}(\omega)=i \sum_{\gamma \delta} \int \frac{d \omega^{\prime}}{2 \pi} G_{\alpha \gamma, \delta \beta}\left(\omega+\omega^{\prime}\right) g_{\delta \gamma}\left(\omega^{\prime}\right),
$$

where the energy dependence is a consequence of the use of the Brueckner $G$ matrix. The latter was computed by solving the Brueckner-Goldstone equation according to Ref. [21] which includes the high-momentum intermediate states that are necessary to the description of SRTC. The Pauli operator used in the calculation of the $G$ matrix excludes all the intermediate states that are part of the model space, in which the LRC are explicitly computed [18]. This doublepartitioning procedure avoids double counting of the $p p$ ladder-diagram contributions to the self-energy. The selfenergy contribution of Eq. (4) is needed in order to generate the correct SP energies for the main shells. Nevertheless, it should be noted that Eq. (4) is expressed in terms of the self-consistent solution $g_{\gamma \delta}(\omega)$. If instead an IPM input is used, the approximated Hartree-Fock contribution may not be sufficient to put the main hole and particle fragments at the right place in energy. Rather, a self-consistent solution of the BHF equations should be employed to evaluate Eq. (4). For example, in Ref. [17], this is done by solving the BHF equations in advance. Then, the set of precomputed SP energies was used in the rest of the calculations. Also, one must note that a relevant contribution to the spin-orbit splitting between the $1 p_{1 / 2}$ and $1 p_{3 / 2}$ shells comes from relativistic effects or three-body forces [31,32]. These contributions are not considered here.

In the present work, this issue was solved by adding an auxiliary one-body term to the BHF self-energy (4). This potential was chosen to be diagonal in the model space basis and it was used to shift the SP energies for the fragments close to the Fermi energy. These corrections were needed during the first iterations, when the IPM starting point was used, and the parameters were fitted to reproduce the correct missing energies for the knockout and the addition of a proton. Once self-consistency is achieved, the BHF term of Eq. (4) generates correctly the contribution to the SP energies that come from two-body interactions. Accordingly, in our calculation the corrections applied to the $s$ and $d$ shells became negligibly small after a few iterations. The $p$ shells continue to require an adjustment of $2.7 \mathrm{MeV}$ for $1 p_{1 / 2}$ and $-0.7 \mathrm{MeV}$ for $1 p_{3 / 2}$, respectively. This is quantitatively in agreement with the need for a contribution from three-body forces as obtained in Ref. [31].

The BHF contribution (4) is also relevant for the normalization of the spectral strengths, since it contributes to the derivative of the self-energy in Eq. (3). In Ref. [17], it was shown that this accounts for a proper treatment of the depletion induced by SRTC at least for the normally occupied shells in the IPM. In the present work, the energy dependence of the BHF contribution was taken into account both in solving the Dyson equation and in the normalization of the spectral amplitudes.

\section{B. Faddeev approach to the self-energy}

In order to compute the last diagrams of Fig. 1, we first consider the polarization propagator describing excited states in the system with $A$ particles 


$$
\begin{aligned}
\Pi_{\alpha \beta, \gamma \delta}(\omega)= & \sum_{n \neq 0} \frac{\left\langle\Psi_{0}^{A}\left|c_{\beta}^{\dagger} c_{\alpha}\right| \Psi_{n}^{A}\right\rangle\left\langle\Psi_{n}^{A}\left|c_{\gamma}^{\dagger} c_{\delta}\right| \Psi_{0}^{A}\right\rangle}{\omega-\left(E_{n}^{A}-E_{0}^{A}\right)+i \eta} \\
& -\sum_{n \neq 0} \frac{\left\langle\Psi_{0}^{A}\left|c_{\gamma}^{\dagger} c_{\delta}\right| \Psi_{n}^{A}\right\rangle\left\langle\Psi_{n}^{A}\left|c_{\beta}^{\dagger} c_{\alpha}\right| \Psi_{0}^{A}\right\rangle}{\omega-\left(E_{0}^{A}-E_{n}^{A}\right)-i \eta}
\end{aligned}
$$

and the two-particle propagator relevant for the $A \pm 2$ excitations

$$
\begin{aligned}
g_{\alpha \beta, \gamma \delta}^{I I}(\omega)= & \sum_{n} \frac{\left\langle\Psi_{0}^{A}\left|c_{\beta} c_{\alpha}\right| \Psi_{n}^{A+2}\right\rangle\left\langle\Psi_{n}^{A+2}\left|c_{\gamma}^{\dagger} c_{\delta}^{\dagger}\right| \Psi_{0}^{A}\right\rangle}{\omega-\left(E_{n}^{A+2}-E_{0}^{A}\right)+i \eta} \\
& -\sum_{k} \frac{\left\langle\Psi_{0}^{A}\left|c_{\gamma}^{\dagger} c_{\delta}^{\dagger}\right| \Psi_{k}^{A-2}\right\rangle\left\langle\Psi_{k}^{A-2}\left|c_{\beta} c_{\alpha}\right| \Psi_{0}^{A}\right\rangle}{\omega-\left(E_{0}^{A}-E_{k}^{A-2}\right)-i \eta} .
\end{aligned}
$$

In their Lehmann representations, these quantities contain all the relevant information regarding $p h$ and $p p(h h)$ collective excitations. The approach of Ref. [26] consists in computing these quantities by solving the $p h$-TDA/RPA and the $p p$-TDA/RPA equations [33], respectively. In the most general case of a fragmented input propagator, the corresponding dressed RPA/TDA (DRPA/DTDA) equations [34,35] are solved. Then, the propagators (5) and (6) have to be coupled to the SP motion to obtain the corresponding approximation for the forward- and backward-going $R(\omega)$ propagators. This is achieved by solving two separate sets of Faddeev equations for the $2 p 1 h$ and the $2 h 1 p$ propagation, respectively.

Taking the $2 p 1 h$ case as an example, one can define three different components $R^{(i)}(\omega) \quad(i=1,2,3)$ that differ from each other by the last interaction that appears in their diagrammatic expansion. These components are solutions of the Faddeev equations [36,37]

$$
\begin{aligned}
R_{\mu \nu \lambda, \alpha \beta \gamma}^{(i)}(\omega)= & \frac{1}{2}\left[G_{\mu \nu \lambda, \alpha \beta \gamma}^{0>}(\omega)-G_{\nu \mu \lambda, \alpha \beta \gamma}^{0>}(\omega)\right] \\
& +G_{\nu \mu \lambda, \mu^{\prime} \nu^{\prime} \lambda^{\prime}}^{0>}(\omega) \Gamma_{\nu^{\prime} \mu^{\prime} \lambda^{\prime}, \mu^{\prime \prime} \nu^{\prime \prime} \lambda^{\prime \prime}}^{(i)}(\omega) \\
& \times\left[R_{\mu^{\prime \prime} \nu^{\prime \prime} \lambda^{\prime \prime}, \alpha \beta \gamma}^{(j)}(\omega)+R_{\mu^{\prime \prime} \nu^{\prime \prime} \lambda^{\prime \prime}, \alpha \beta \gamma}^{(k)}(\omega)\right],
\end{aligned}
$$

in which repeated indices are summed over and $(i, j, k)$ are cyclic permutations of $(1,2,3)$. In Eq. $(7), G^{0>}(\omega)$ is the forward-going part of the $2 p 1 h$ propagator for three noninteracting lines. Using the notation introduced in Eq. (1), we have

$$
G_{\mu \nu \lambda, \alpha \beta \gamma}^{0>}(\omega)=\sum_{n_{1}, n_{2}, k} \frac{\left(\mathcal{X}_{\mu}^{n_{1}} \mathcal{X}_{\nu}^{n_{2}} \mathcal{Y}_{\lambda}^{k}\right) * \mathcal{X}_{\alpha}^{n_{1}} \mathcal{X}_{\beta}^{n_{2}} \mathcal{Y}_{\gamma}^{k}}{\omega-\left(\varepsilon_{n_{1}}^{+}+\varepsilon_{n_{2}}^{+}-\varepsilon_{k}^{-}\right)+i \eta}
$$

The Faddeev vertices $\Gamma^{(i)}(\omega)$ contain the couplings of a $p h$ or $p p(h h)$ collective excitation and a freely propagating line. A well known characteristic of the Faddeev formalism is that it introduces spurious solutions in addition to the correct eigenstates of the Schrödinger equation [38]. In Ref. [26] it was shown that this issue imposes constraints on the choice
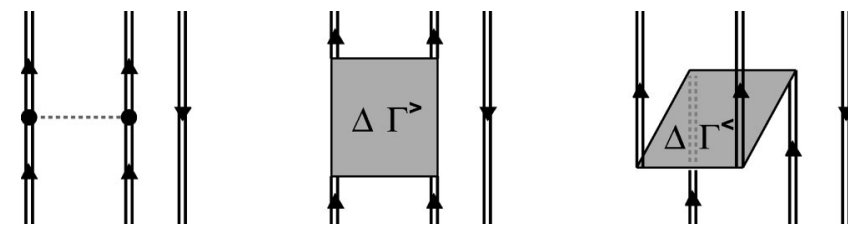

FIG. 2. Faddeev vertex for the coupling of a $p p$ excitation to a hole line. Here $\Delta \Gamma^{>}$and $\Delta \Gamma^{<}$represent the forward- and backward-going part of the $p p$ DRPA propagator.

of the vertex $\Gamma^{(i)}(\omega)$ terms and as a consequence both the forward- and backward-going parts of the collective RPA excitations should be included in these terms. Figure 2 shows an example of the diagrammatic expansion of the vertex corresponding to attaching a hole line to a $p p(h h)$ phonon. By summing over all the Faddeev components [and subtracting $G^{0>}(\omega)$ to avoid double counting] one finally obtains the $2 p 1 h$ propagator

$$
\begin{aligned}
R_{\mu \nu \lambda, \alpha \beta \gamma}^{(2 p 1 h)}(\omega)= & \sum_{i=1,2,3} R_{\mu \nu \lambda, \alpha \beta \gamma}^{(i)}(\omega)-\frac{1}{2}\left[G_{\mu \nu \lambda, \alpha \beta \gamma}^{0>}(\omega)\right. \\
& \left.-G_{\nu \mu \lambda, \alpha \beta \gamma}^{0>}(\omega)\right],
\end{aligned}
$$

from which the self-energy can be easily derived. The above consideration apply to the $2 h 1 p$ propagation as well, for which a set of equations analogous to Eqs. (7), (8), and (9) is employed.

The actual application of the Faddeev formalism to $2 p 1 h / 2 h 1 p$ propagation involves a certain number of complications. These require a slight redefinition of the components $R^{(i)}(\omega)$ introduced above and a rearrangement of Eq. (7). Eventually, one is left with an eigenvalue problem that can be projected on a Hilbert space that spans only the correct physical solutions. The details of these issues have already been presented in Ref. [26] and will not be discussed any further in this paper. The important thing to note here is that this formalism allows the inclusion of the effects of $p h$ and $p p(h h)$ motion not only at the TDA level but also at the more collective RPA level. These excitations are coupled to each other by the Faddeev equations, generating diagrams similar to the one shown in Fig. 3. This also assures that Pauli correlations are properly taken into account at the $2 p 1 h / 2 h 1 p$ level. In addition, one can employ dressed SP propagators in these equations to generate a self-consistent solution.

\section{Application to ${ }^{16} \mathrm{O}$ and iterative procedure}

In the calculations described below, the Dyson equation was solved within a model space consisting of harmonic oscillator SP states. An oscillator parameter $b=1.76 \mathrm{fm}$ was chosen and all the first four major shells (from $1 s$ to $2 p 1 f$ ) plus the $1 g_{9 / 2}$ where included. Inside this model space, the interaction used was a Brueckner $G$ matrix [21] derived from the Bonn-C potential [39]. The results of Refs. [40,41], suggest that this model space is large enough to properly account for the low-energy collective states that we are mainly interested in, the SRTC being accounted for through the 


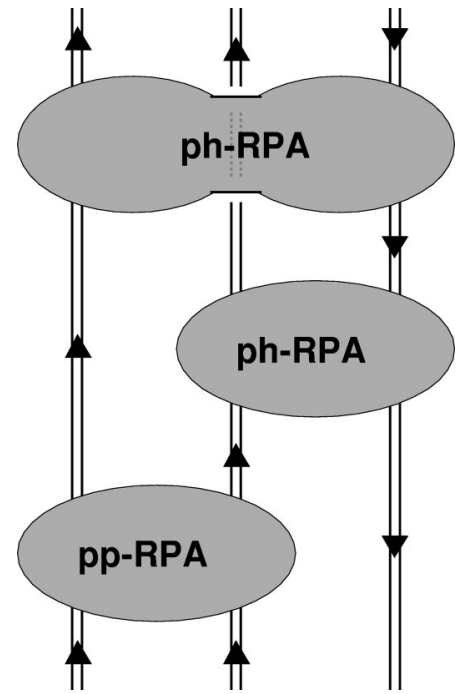

FIG. 3. Example of diagrams that are summed to all orders by means of the Faddeev equations.

$G$-matrix effective interaction. These calculations also show that screening effects, such as those included by dressing the SP propagator, improve the convergence of results with respect to the dimension of the model space. The Bonn-C potential does not include any charge independence breaking term and the Coulomb interaction between protons was not taken into account. Therefore the same results were obtained for both neutron and proton spectral functions. As mentioned above the energy dependence of the $G$ matrix was completely taken into account in the evaluation of the BHF part of the self-energy and in computing the normalization of spectral amplitudes. For the solution of the Faddeev equations a $G$ matrix evaluated at a fixed starting energy was employed. In this case a value of $-25 \mathrm{MeV}$ has been chosen as a suitable average of the most important $2 h 1 p$ states which are of interest here.

By using an IPM propagator as input Green's function, the BHF term (4) was computed and the Faddeev equations were solved to obtain the irreducible self-energy of Fig. 1. From the solution of Eq. (2) the SP spectral functions were obtained. The solution to the Dyson equation (2) contains a large number of fragments, most of which are quite small. A fully self-consistent solution requires a method in which the SP strength is binned over a large energy domain [22]. The number of poles and the resulting $2 p 1 h$ and $2 h 1 p$ are then too numerous for a practical solution of the Faddeev equations. To obtain some insight into the effects of selfconsistency we have chosen the following procedure to account for the fragmentation. For SP levels far from the Fermi energy, we have kept two poles above and below the Fermi energy, except for the $f$ and $g$ shells for which only one effective hole pole was kept. For the levels near the Fermi energy, the quasiparticle (hole) fragment was kept, including its location and strength. When two effective poles on one side of the Fermi energy were included, the fragment closest to the Fermi energy was kept with its strength and the rest of the strength was collected at a location determined by weighing the remaining fragment energies with the corresponding strength. The resulting dressed propagator still contains all the relevant low-energy fragmentation and at the same time it has a number of poles small enough to be used as input in another Faddeev DRPA calculation.

For too attractive interactions, the RPA approach can give rise to instabilities. This situation is particularly critical for the isoscalar $0^{+}$channel [24]. In performing our calculations with a dressed propagator, the $p h$ DRPA equations gave an unstable solution for the first $0^{+}$excited state of ${ }^{16} \mathrm{O}$. Naturally, the instability of the lowest $p h 0^{+}$state tends to disappear when a more negative starting energy is chosen for the $G$ matrix since such a choice reduces the attraction in this channel. Since the first $0^{+}$state is of particular importance, we decided not to discard it but to compute it in a regime were the instability disappears, adopting the following prescription. A stable solution for the spectroscopic amplitudes of this state was obtained by solving the $p h$ DPRA equations with a $G$ matrix at a starting energy of $-110 \mathrm{MeV}$. The energy of the state was then kept fixed at the experimental energy of $6.05 \mathrm{MeV}$. The solution obtained, was then substituted for the unstable one. All the remaining $0^{+}$levels were properly computed with a $G$ matrix at $-25 \mathrm{MeV}$.

Obviously, such a prescription is somewhat artificial and should be avoided in future calculations. When improved phonons are considered, the RPA equations tend to yield stable results and allow for more attractive interactions [41]. Such improvement of the phonon calculation will therefore eliminate the need for this artificial step. This will be the topic of future work [43].

\section{RESULTS FOR THE ONE-HOLE SPECTRAL FUNCTION}

\section{A. Effects of RPA correlations}

By using an IPM ansatz as input propagator, the Faddeev equations have been applied to obtain the self-energy of ${ }^{16} \mathrm{O}$ in both TDA and RPA approximations. The resulting selfenergy was then used in the Dyson equation (2) to obtain the SP spectral functions for the removal (one-hole) and addition (one-particle) of a nucleon. The values of the spectroscopic factors for the main particle and hole shells close to the Fermi energy are reported in Table I. The hole strengths given by TDA are 0.775 for $p_{1 / 2}$ and 0.766 for $p_{3 / 2}$, in close agreement with the results of Ref. [17] (to which the present TDA calculation is equivalent). The introduction of RPA correlations reduces these values and brings them down to 0.745 and 0.725 , respectively. This result reduces the discrepancy with the experiment by about $4 \%$ and shows that collectivity beyond the TDA level is relevant to explain the quenching of spectroscopic factors. Since the present formalism does not account for center-of-mass effects, the above quantities need to be increased by about $7 \%$ before they are compared with the experiment $[15,16]$. It should be noted that the present RPA results describing the $p h$ and $p p(h h)$ spectrum suffer from the usual problems associated with RPA. One such feature, as already noted above for the $p h 0^{+}$state, is the appearance of at most one collective state for a given $J^{\pi}$, whereas many low-lying isoscalar natural parity states are observed experimentally. This feature implies that especially 
TABLE I. Spectroscopic factors for ${ }^{16} \mathrm{O}$ as computed in both TDA and RPA schemes using an IPM input. Listed are the strengths of the main (particle or hole) fragments for the five levels close to the Fermi energy. All values are given as a fraction of the corresponding IPM value.

\begin{tabular}{ccc}
\hline \hline Shell & TDA & RPA \\
\hline$d_{3 / 2}$ & 0.866 & 0.838 \\
$s_{1 / 2}$ & 0.882 & 0.842 \\
$d_{5 / 2}$ & 0.894 & 0.875 \\
$p_{1 / 2}$ & 0.775 & 0.745 \\
$p_{3 / 2}$ & 0.766 & 0.725 \\
\hline \hline
\end{tabular}

the $p h$ spectrum does not provide a very good description of the experimental data. One may therefore hope that further improvements of the description of the RPA phonons themselves will close the gap with the experimental data further.

Together with the main fragments, the Dyson equation produces also a large number of solutions with small spectroscopic factors. For the one-hole spectral function, this strength extends down to about $-130 \mathrm{MeV}$. This background partly represents the strength that is removed from the main peaks and shifted up to medium missing energies. The energy dependence of the $G$ matrix accounts for another $10 \%$ effect in pushing the strength of the mostly occupied shells to high energies in the particle domain. We note that the location of this strength cannot be explicitly calculated in the present approach but corresponds to very large energies [1]. The occupation number coming from both the background contribution at negative energies, as well as the main hole fragments is displayed in Table II for the most important shells. Summing these numbers together with the occupation of the main peaks and weighing them by a factor of $2(2 j$ +1 ), one gets a total number of particles equal to about 15 nucleons. This violation of particle number is a consequence of the energy dependence of the $G$ matrix. The remaining strength is then accounted for by the presence of highmomentum components due to SRTC not explicitly calculated in the present scheme. The present result therefore also gives an estimate of the number of these high-momentum particles that are shifted to even higher energies (more negative). These high-momentum components are included in the results of Refs. [12,42] and their strength corresponds to the number of missing nucleons in the present calculation. The effects of SRTC on the reduction of quasihole spectroscopic factors are properly included, through the energy dependence of the $G$ matrix interaction.

Figure 4 displays the TDA and RPA one-hole spectral function for the $p_{1 / 2}$ and $p_{3 / 2}$ states. In this figure the theoretical spectral function is binned in order to make a comparison with the experimental results. These results demonstrate that neither of the two approaches explains the breaking of the main $p_{3 / 2}$ peak when an input IPM propagator is used. The main difference between the two results are the $4 \%$ smaller peaks obtained in the RPA approach. The results for positive parity shells are shown in Fig. 5. The solid bars refer to results for orbital angular momentum $l$ $=2$ and the open bars to $l=0$, respectively. We observe that
TABLE II. Occupation and depletion numbers for the most relevant shells of ${ }^{16} \mathrm{O}$ as computed in both TDA and RPA schemes using an IPM input. All the results are given as a fraction of the corresponding IPM value. Also shown is the result for the total number of nucleons obtained by summing over all the hole fragments.

\begin{tabular}{lcccc}
\hline \hline & \multicolumn{2}{c}{ TDA } & \multicolumn{2}{c}{ RPA } \\
Shell & Particle & Hole & Particle & Hole \\
\hline $2 p_{1 / 2}$ & 0.983 & 0.014 & 0.980 & 0.017 \\
$2 p_{3 / 2}$ & 0.980 & 0.016 & 0.978 & 0.018 \\
$1 d_{3 / 2}$ & 0.958 & 0.038 & 0.945 & 0.051 \\
$2 s_{1 / 2}$ & 0.954 & 0.039 & 0.916 & 0.074 \\
$1 d_{5 / 2}$ & 0.961 & 0.035 & 0.946 & 0.049 \\
$1 p_{1 / 2}$ & 0.102 & 0.828 & 0.128 & 0.804 \\
$1 p_{3 / 2}$ & 0.076 & 0.856 & 0.107 & 0.828 \\
$1 s_{1 / 2}$ & 0.044 & 0.888 & 0.057 & 0.876 \\
\hline Total occ. & \multicolumn{5}{|c}{15.06} \\
\hline \hline
\end{tabular}

the RPA approach generates two hole peaks with angular momenta $d_{5 / 2}$ and $s_{1 / 2}$ at small missing energy. These peaks are found separated from the rest of strength at -15.6 and $-15.8 \mathrm{MeV}$, respectively, which differ from the experimental value by about $2 \mathrm{MeV}$. The theory also predicts a spectroscopic factor of $0.1 \%$ for $d_{5 / 2}$ which is smaller than the experimental value of $1.9 \%$. This represents an improvement with respect to the TDA, where such a fragment is not reproduced at all. The agreement is better with the $s_{1 / 2}$ fragment for which the theory predicts $3.0 \%$ and the experimental value is $1.8 \%$. At energies below $-20 \mathrm{MeV}$, the experimental $s_{1 / 2}$ strength is distributed almost continuously and increases as the energy approaches the region corresponding to giant resonances. In the present calculation, based on a finite number of discrete states, the theory predicts a fragmentation over fewer isolated peaks with higher spectroscopic strengths.

\section{B. Effects of fragmentation}

The RPA results were iterated a few times, with the aim of studying the effects of fragmentation on the RPA phonons and, subsequently, on the spectral strength. This was done by employing the prescription for representing the strength distribution with effective poles that is described in Sec. II C. The negative parity hole spectral function resulting from the third iteration is shown in the lower panel of Fig. 4. The main difference between these results and the one obtained by using an IPM input is the appearance of a second smaller $p_{3 / 2}$ fragment at $-26.3 \mathrm{MeV}$. This peak rises in the first two iterations and appears to become stable in the last one, with a spectroscopic factor of $2.6 \%$. This can be interpreted as a peak that describes the fragments seen experimentally at slightly lower energy. This is the first time that such a fragment is obtained in calculations of the spectral strength. Further insight into the appearance of this strength is discussed in Sec. III C.

A second effect of including fragmentation in the construction of the RPA phonons is to increase the strength of 


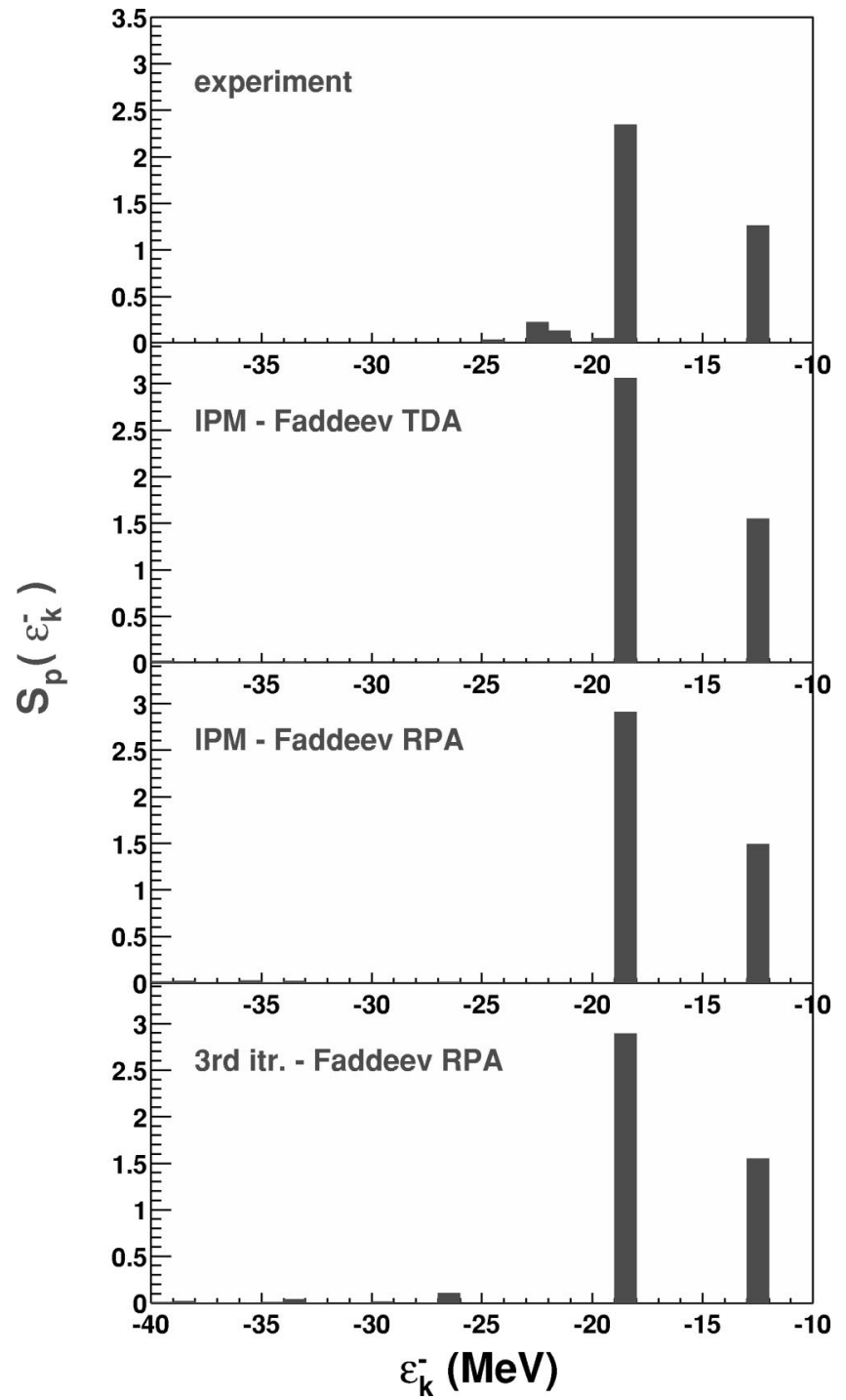

FIG. 4. One-proton removal strength as a function of the hole SP energy $\varepsilon_{k}^{-}=E_{0}^{A}-E_{k}^{A-1}$ for ${ }^{16} \mathrm{O}$ and angular momentum $l=1$. The experimental values are taken from Ref. [10]. The theoretical results have been calculated in both TDA and RPA approximation with an IPM model input. The bottom panel includes the results of iterating the fragmentation pattern through the construction of DRPA phonons.

the main hole peaks. The spectroscopic factors for the main $p$ peaks, as obtained from different iterations, are reported in Table III. The $p_{1 / 2}$ strength increases from the 0.745 obtained with IPM input to 0.774 , essentially cancelling the improvement gained by the introduction of RPA correlations over the TDA ones. The main peak of the $p_{3 / 2}$ remains at 0.722 but the appearance of the secondary fragment slightly increases the overall strength at low energy also. This behavior can be understood by realizing that with an IPM input most of the phonons are somewhat more collective than the ones obtained from employing dressed propagators with the exception of the special case of the $p h 0^{+}$. As a result, one can expect a reduced effect of RPA correlations when fragmentation is included in the construction of the phonons. This

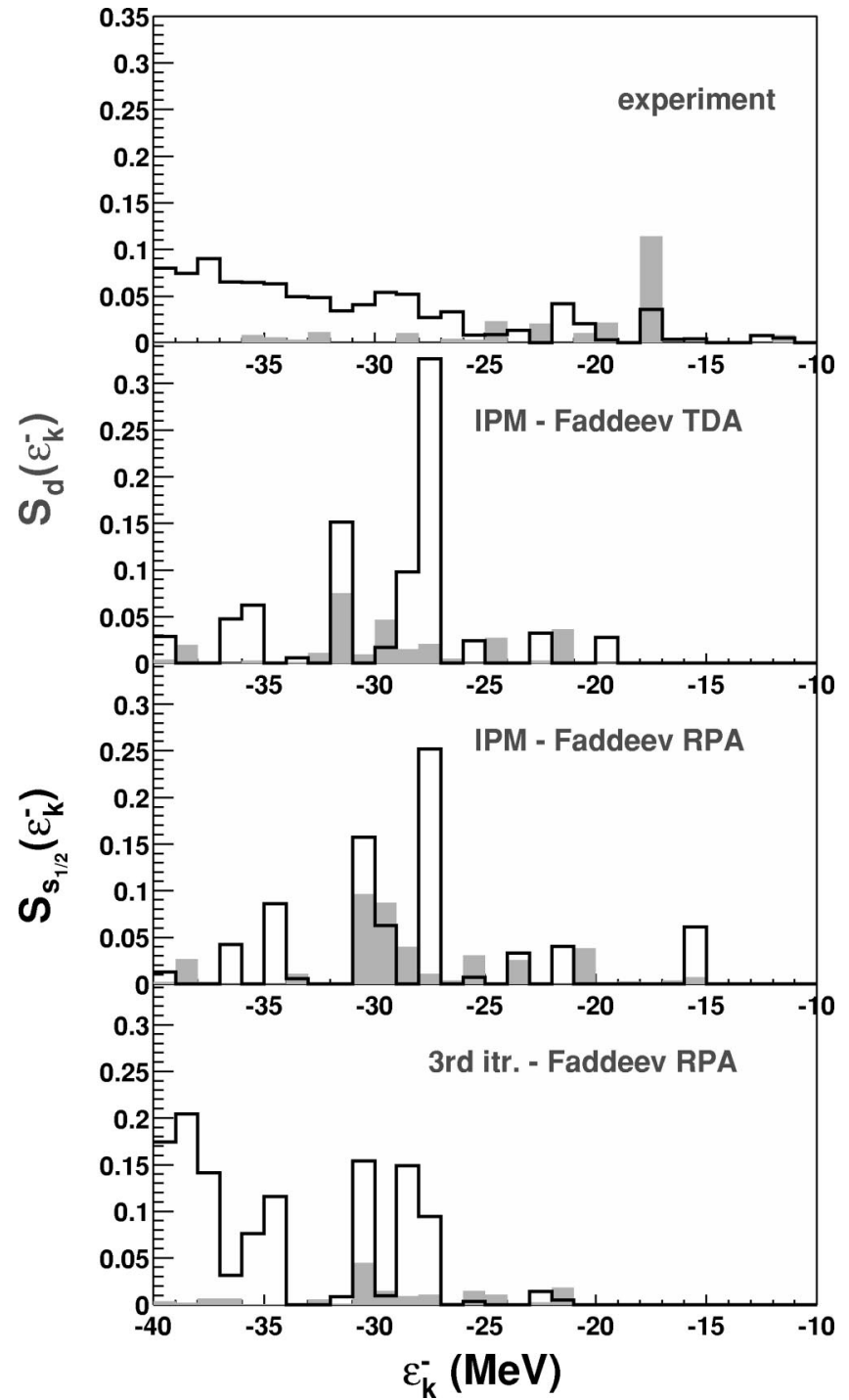

FIG. 5. One-proton removal strength as a function of the hole SP energy $\varepsilon_{k}^{-}=E_{0}^{A}-E_{k}^{A-1}$ for ${ }^{16} \mathrm{O}$ and positive parity final states. The solid bars correspond to results for orbital angular momentum $l=2$, while the thick lines refer to $l=0$. The experimental values are taken from Ref. [10]. The theoretical results have been calculated in both TDA and RPA approximation with an IPM model input. The bottom panel includes the effect of fragmentation on the construction of the DRPA phonons after three iterations.

feature has also been observed in other self-consistent calculations of the SP spectral strength, for example in nuclear matter [44]. Obviously, this makes the disagreement with experiments a little worse and additional work is needed to resolve the disagreement with the data. Nevertheless it is clear that fragmentation is a relevant feature of nuclear systems and that it has to be properly taken into account. It is also worth noting that already after a few iterations, all the main quantities of Table III tend to stabilize and sustain themselves in a self-consistent way.

Table III also shows the total number of particles obtained at each iteration (derived by summing over the hole strength). This result corresponds to about 14.6 nucleons 
TABLE III. Hole spectroscopic factors $\left(Z_{\alpha}\right)$ for knockout of a $l=1$ proton from ${ }^{16} \mathrm{O}$ and occupation numbers $\left(n_{\alpha}\right)$ for different angular momenta of the nucleon. These results refer to the first three iterations of the DRPA equations. All the values are given as a fraction of the corresponding IPM value and in the case of $l=0$ and $l=1$ are summed over the principal h.o. quantum numbers belonging to the model space (i.e., $s_{1 / 2}$ stands for the sum of $1 s_{1 / 2}$ and $2 s_{1 / 2}$, similary for $p_{1 / 2}$ and $p_{3 / 2}$ ). Also included is the total number of nucleons for each iteration.

\begin{tabular}{lccc}
\hline \hline Shell & 1st itr. & 2nd itr. & 3rd itr. \\
\hline$Z_{p_{1 / 2}}$ & 0.775 & 0.777 & 0.774 \\
$Z_{p_{3 / 2}}$ & & & \\
& 0.725 & 0.727 & 0.722 \\
$n_{d_{3 / 2}}$ & 0.015 & 0.027 & 0.026 \\
$n_{d_{5 / 2}}$ & 0.025 & 0.025 & 0.026 \\
$n_{p_{1 / 2}}$ & 0.020 & 0.021 & 0.021 \\
$n_{p_{3 / 2}}$ & 0.850 & 0.848 & 0.848 \\
$n_{s_{1 / 2}}$ & 0.870 & 0.871 & 0.870 \\
\hline Total occ. & 0.911 & 0.914 & 0.916 \\
\hline \hline
\end{tabular}

when fragmentation is included. This gives an estimate for the overall occupancy of high momentum states of about $10 \%$, in agreement with direct calculations of SRTC $[12,42]$. We observe that this estimate is different from the results for the IPM input quoted in Table II. We associate this difference with the energy dependence of the $G$ matrix which is sampled differently in both cases. In the IPM calculation the lowest three shells are included at the harmonic oscillator level. Upon iteration, which involves the changing BHF contribution, the admixture of the other $s$ and $p$ shells is included and will generate a slightly different effect related to the energy dependence of the $G$ matrix since different matrix elements are sampled in each case. We note here that for this reason there is also a distinct difference between the quasiparticle and quasihole strengths near the Fermi energy as shown in Table I of about $10 \%$. This same difference appears in the summed strengths appearing in Table II. In both cases there appears more strength in the particle domain than is appropriate for the effect of SRTC. In the present approach we cannot treat this effect for particle shells properly since the $G$ matrix of Ref. [21] is constructed only for negative energy. The derivative of the energy dependence of the $G$ matrix at energies relevant for particle states will therefore not reflect the true depletion due to SRTC. For this reason the summed strength for the particle states is close to 1 in Table II.

The results of the third iteration are also given in Fig. 5 for the relevant positive parity spectral functions. We note that the $s_{1 / 2}$ and $d_{5 / 2}$ hole fragments at $-15 \mathrm{MeV}$ are no longer generated by these iterated calculations. Also, as a consequence of dressing the input propagator, more poles are produced as solutions of the Dyson equation (2). This allows for a better distribution of the $s_{1 / 2}$ strength at medium missing energies. Similar results have been obtained in the selfconsistent second-order calculation for ${ }^{48} \mathrm{Ca}$ in Ref. [22].
Presumably, a more complete representation of the strength in the input propagator would further improve the $l=0$ strength distribution.

\section{Role of $0^{+}$and $3^{-}$excited states in ${ }^{16} \mathrm{O}$}

A deeper insight into the mechanisms that generate the fragmentation pattern can be gained by looking directly at the connection between the spectral function and some specific collective states. To clarify this point we repeated the third iteration using exactly the same input but without replacing the unstable $p h 0^{+}$state, which was instead discarded. The resulting $p$ hole spectral function is shown in the upper panel of Fig. 6. In this calculation no breaking of the $p_{3 / 2}$ peak is obtained. Instead a single peak is found with a spectroscopic factor equal to 0.75 which corresponds to the sum of the two fragments that are obtained when the $0^{+}$state is taken into account. This result can be interpreted by considering the $p_{3 / 2}$ fragments as a hole on the ground state and on the first excited $0^{+}$state of the ${ }^{16} \mathrm{O}$ core, respectively. If the latter two levels are close enough to each other in the calculation, a mixing between the two configurations can occur and a second smaller fragment is generated. When the excited $0^{+}$state is removed from the calculation or, as in the TDA approach, is found far above the experimental energy, the calculation can reproduce only one single peak. Obviously, it is understood from the dressed results of Fig. 4 that further improvements have to be made in other to describe properly the strength and the missing energy of the smaller fragments. A candidate to consider in this improvement is the role of the first $2^{+}$state in ${ }^{16} \mathrm{O}$, which can also couple to generate $p_{3 / 2}$ hole fragments but that was not included here since it cannot be obtained by the present $p h$ DRPA calculation, at least not at low enough energy.

The other two low-lying states of ${ }^{16} \mathrm{O}$ that may be of some relevance are the isoscalar $1^{-}$and $3^{-}$. These excitation are reproduced reasonably well by RPA type calculations [24] but are typically found at higher energies than the experimental ones. In the present case the third iteration gives 9.4 and $10.8 \mathrm{MeV}$ for $3^{-}$and $1^{-}$, respectively, which is about $3 \mathrm{MeV}$ above the experimental results. This points to a need for a more attractive $G$-matrix interaction. We mention here that the present $G$ matrix is calculated without any binding correction of the SP energies for particle states which could have some influence on the strength of the effective interaction. The lower panels of Fig. 6 show the results for the even parity spectral functions that are obtained if the $3^{-}$ alone or both $3^{-}$and $1^{-}$are shifted down to their experimental values. In this case, a $d_{5 / 2}$ hole peak is obtained at low missing energy. This result is also quantitatively more satisfactory than what was obtained in the RPA calculation based on the IPM, since in this case it is found at -17.7 $\mathrm{MeV}$ (in agreement with experiment) and with a spectroscopic strength of $0.5 \%$. It is interesting to note that the shifting of the $3^{-}$collective state does not produce any other noticeable change in the theoretical spectral function. The same applies if also the $1^{-}$is shifted. 

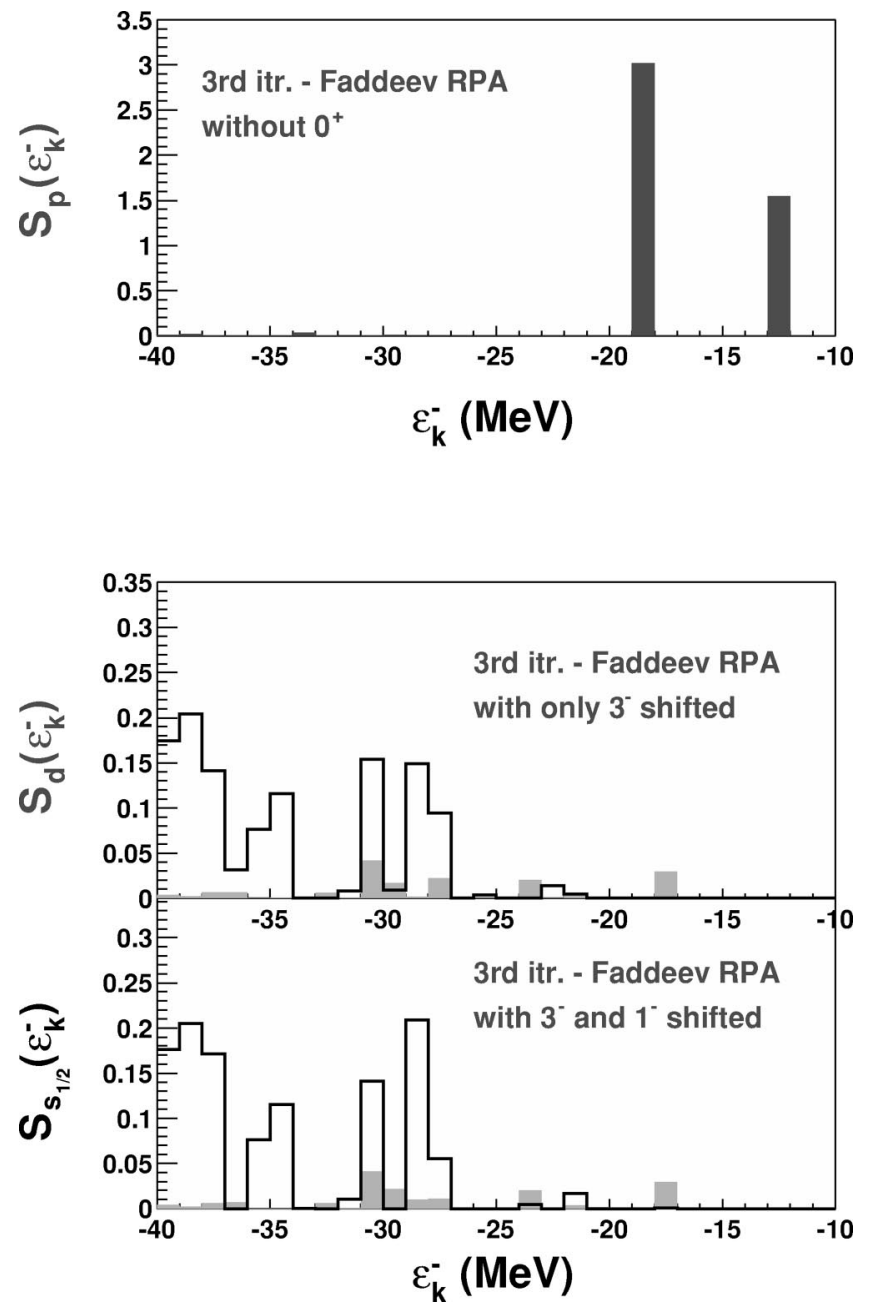

FIG. 6. One-proton removal strength resulting from repeating the third iteration with a modified $p h$ propagator. The upper panel refers to the results for $l=1$ when the lowest $0^{+}$state of ${ }^{16} \mathrm{O}$ is excluded. The lower panels give the $l=0$ (thick curve) and $l=2$ (solid bar) results obtained when the $3^{-}$alone or both the $3^{-}$and $1^{-}$states are shifted to their experimental energies.

It appears therefore that the main impediment for further improvements of the description of the experimental data is associated with the deficiencies of the RPA (DRPA) description of the excited states. One important problem is the appearance of at most one collective phonon for a given $J^{\pi}, T$ combination. Experimentally, several low-lying isoscalar $0^{+}$ and $2^{+}$excited states are observed at low energy in ${ }^{16} \mathrm{O}$ as well as additional $3^{-}$and $1^{-}$states. A possible way to proceed would be to first concentrate on an improved description of the collective phonons by extending the RPA to explicitly include the coupling to two-particle-two-hole $(2 p 2 h)$ states. Such an extended RPA procedure has been applied in heavier nuclei with considerable success [40,41]. In order to be relevant for ${ }^{16} \mathrm{O}$, this approach requires an extension in which the coherence of the $2 p 2 h$ states is included in the form of the presence of two phonon excitations. Such contributions arise naturally when the response is calculated by using the Baym-Kadanoff construction of the irreducible $p h$ interaction which is based on a self-consistent treatment of the self-energy [45].

\section{CONCLUSIONS}

In the present paper the Faddeev technique has been applied to study $2 h 1 p$ correlations at small missing energies for the nucleus of ${ }^{16} \mathrm{O}$. The application of the Faddeev method allows for the first time the treatment of the coupling of $p h$ and $h h$ collective modes within an RPA framework and to all orders in the nucleon self-energy. The resulting spectral function shows better agreement with experimental data than all previous calculations. Additional encouraging results are obtained in the form low-lying positive parity fragments.

These results were extended by recalculating the RPA phonons using the so-obtained fragmented SP propagator. The inclusion of this fragmentation in the phonons leads to the appearance of an additional $p_{3 / 2}$ fragment at low energy in agreement with experiment. Other features, such as the presence of positive parity $5 / 2$ and $1 / 2$ holes at $-17 \mathrm{MeV}$, cannot be obtained when the present calculations are iterated.

We have further identified the important role played by the low-lying $p h$ states of ${ }^{16} \mathrm{O}$. The low-lying $0^{+}$appears to be at least partially responsible for the splitting of the $p_{3 / 2}$ strength at low energy, whereas the low-lying $3^{-}$state plays a decisive role in the presence of $d_{5 / 2}$ strength at low energy. The results of the present calculations are therefore very sensitive to the quality of the RPA (DRPA) description of the $p h$ spectrum. It is well known that this description is as yet unsatisfactory but key ingredients for further improvements can be identified through the Baym-Kadanoff procedure based on self-consistent propagators. We therefore conclude that the present results show that further improvement in the understanding of the low-energy fragmentation can be gained. To do this, the employed approximations need to correctly reproduce all the lowest $p h$ collective modes. We therefore propose to first improve the quality of the RPA phonons before engaging in a fully self-consistent evaluation of the one- and two-hole spectral functions for ${ }^{16} \mathrm{O}$ [43].

\section{ACKNOWLEDGMENTS}

Part of the calculations described in this paper were performed on the SGI Origin 2000 of the Center for Scientific Parallel Computing of Washington University, St. Louis. One of us (C.B.) would like to acknowledge the hospitality of the Institut für Theoretische Physik at the University of Tübingen, where this work was completed. This work was supported by the U.S. National Science Foundation under Grant No. PHY-9900713. 
[1] B. E. Vonderfecht, W. H. Dickhoff, A. Polls, and A. Ramos, Phys. Rev. C 44, R1265 (1991).

[2] W. H. Dickhoff and H. Müther, Rep. Prog. Phys. 55, 1947 (1992).

[3] G. A. Rijsdijk, K. Allaart, and W. H. Dickhoff, Nucl. Phys. A550, 159 (1992).

[4] A. E. L. Dieperink and P. de Witt Huberts, Annu. Rev. Nucl. Part. Sci. 40, 239 (1990).

[5] I. Sick and P. de Witt Huberts, Comments Nucl. Part. Phys. 20, 177 (1991).

[6] L. Lapikás, Nucl. Phys. A553, 297c (1993).

[7] C. J. C. Onderwater et al., Phys. Rev. Lett. 78, 4893 (1997).

[8] C. J. C. Onderwater et al., Phys. Rev. Lett. 81, 2213 (1998).

[9] G. Rosner, Prog. Part. Nucl. Phys. 44, 99 (2000).

[10] M. Leuschner et al., Phys. Rev. C 49, 955 (1994).

[11] J. M. Udías, J. A. Caballero, E. Moya de Guerra, J. R. Vignote, and A. Escuderos, Phys. Rev. C 64, 024614 (2001).

[12] H. Müther and W. H. Dickhoff, Phys. Rev. C 49, R17 (1994).

[13] M. Radici, S. Boffi, S. C. Pieper, and V. R. Pandharipande, Phys. Rev. C 50, 3010 (1994).

[14] A. Fabrocini and G. Co', Phys. Rev. C 63, 044319 (2001).

[15] A. E. L. Dieperink and T. de Forest, Jr., Phys. Rev. C 10, 543 (1974).

[16] D. Van Neck, M. Waroquier, A. E. L. Dieperink, S. C. Pieper, and V. R. Pandharipande, Phys. Rev. C 57, 2308 (1998).

[17] W. J. W. Geurts, K. Allaart, W. H. Dickhoff, and H. Müther, Phys. Rev. C 53, 2207 (1996).

[18] K. Amir-Azimi-Nili, H. Müther, L. D. Skouras, and A. Polls, Nucl. Phys. A604, 245 (1996).

[19] W. J. W. Geurts, K. Allaart, W. H. Dickhoff, and H. Müther, Phys. Rev. C 54, 1144 (1996).

[20] C. Giusti, F. D. Pacati, K. Allaart, W. J. W. Geurts, W. H. Dickhoff, and H. Müther, Phys. Rev. C 57, 1691 (1998).

[21] H. Müther and P. Sauer, in Computational Nuclear Physics, edited by K.-H. Langanke et al. (Springer, Berlin, 1993).

[22] D. Van Neck, M. Waroquier, and J. Ryckebusch, Nucl. Phys. A530, 347 (1991).

[23] G. A. Rijsdijk, W. J. W. Geurts, K. Allaart, and W. H. Dickhoff, Phys. Rev. C 53, 201 (1996).

[24] P. Czerski, W. H. Dickhoff, A. Faessler, and H. Müther, Phys. Rev. C 33, 1753 (1986).

[25] W. H. Dickhoff, in Condensed Matter Theories, edited by J. S.
Arponen, R. F. Bishop, and M. Manninen (Plenum, New York, 1988), Vol. 3, p. 261.

[26] C. Barbieri and W. H. Dickhoff, Phys. Rev. C 63, 034313 (2001).

[27] C. Barbieri and W. H. Dickhoff, in Proceedings of the Fifth Workshop on Electromagnetically Induced Two-Hadron Emission, edited by P. Grabmyar et al. (University of Lund, published on CD, ISBN: 91-631-1612-X, 2001), p. 108.

[28] A. L. Fetter and J. D. Walecka, Quantum Theory of ManyParticle Physics (McGraw-Hill, New York, 1971).

[29] A. A. Abrikosov, L. P. Gorkov, and I. E. Dzyaloshinski, Methods of Quantum Field Theory in Statistical Physics (Dover, New York, 1975).

[30] J. Winter, Nucl. Phys. A194, 535 (1972).

[31] S. C. Pieper and V. R. Pandharipande, Phys. Rev. C 70, 2541 (1993).

[32] R. Brockmann, Phys. Rev. C 18, 1510 (1978); H. Müther, R. Machleidt, and R. Brockmann, ibid. 42, 1981 (1990).

[33] P. Ring and P. Schuck, The Nuclear Many-Body Problem (Springer, New York, 1980).

[34] G. A. Rijsdijk, W. J. W. Geurts, M. G. E. Brand, K. Allaart, and W. H. Dickhoff, Phys. Rev. C 48, 1752 (1993).

[35] W. J. W. Geurts, K. Allaart, and W. H. Dickhoff, Phys. Rev. C 50, 514 (1994).

[36] L. D. Faddeev, Zh. Éksp. Teor. Fiz. 391459 (1961) [Sov. Phys. JETP 12, 1014 (1961)].

[37] W. Glöckle, The Quantum Mechanical Few-Body Problem (Springer, Berlin, 1983).

[38] P. Navrátil, B. R. Barrett, and W. Glöckle, Phys. Rev. C 59, 611 (1999), and references therein.

[39] R. Machleidt, Adv. Nucl. Phys. 19, 191 (1989).

[40] M. G. E. Brand, K. Allaart, and W. H. Dickhoff, Phys. Lett. B 214, 483 (1988).

[41] M. G. E. Brand, K. Allaart, and W. H. Dickhoff, Nucl. Phys. A509, 1 (1990).

[42] A. Polls, H. Müther, and W. H. Dickhoff, Phys. Rev. C 51, 3040 (1995).

[43] C. Barbieri, Ph.D. thesis, Washington University, St. Louis, 2002.

[44] E. P. Roth, Ph.D. thesis, Washington University, St. Louis, 2000.

[45] G. Baym and L. P. Kadanoff, Phys. Rev. 124, 287 (1961). 